\title{
VARICOSE VEINS
}

\section{Some Comments on the Repair of Surgical Failures}

\author{
By R. Rowden Foote
}

Surgeon in Charge of Varicose Vein Department, Harrow Hospital

There is no operation which is capable of restoring a normal physiology to a limb which is the seat of varicose disease. So, in actual fact, no form of surgical approach is capable of producing perfection. The word 'failure,' when applied to the results of surgery, must, for the purposes of this article, be used only when the diseased limb is either not improved or has become worse since operation.

Varicose disease is a progressive condition showing a steady deterioration over the years. For this reason the prognosis regarding cure is better amongst our older patients, who will obviously need the use of their legs for a shorter time than will those who are their juniors. Again, surgical success is more noticeable in those cases presenting a marked incompetence of the valves of the superficial venous system associated with a gross dilatation and tortuosity of the affected veins. It has been said by others that before considering surgery for a gastric ulcer the patient ' has to earn his operation.' This remark can be applied with equal truth to the sufferer from varicose disease. If this fact be remembered, mild cases will not be submitted to unnecessary surgery performed purely from the cosmetic angle and we shall have fewer failures with which to contend.

There has been an unremitting struggle by surgeons throughout the ages against the almost uncanny persistence of varicose disease, and those who care to read will find great interest in studying the varied angles of attack which have been levelled against the varix since the days when Hippocrates practised acu-puncture and Galen, a hundred or so years later, tore these pernicious weeds from the limb by means of a hook. The first glimmer of the development of our modern surgical attack directed at the roots of the internal saphenous vein was given to us by Paul of Aegina who, some thirteen hundred years ago, recognized a sufficiency regarding the anatomy of 'the long saphenous vein and its tributaries to secure, as he thought, the main trunk from which all the branches arise. These great men of the past all failed to produce a cure, and it is doubtful whether, in most cases, they produced an improvement. As a result, throughout the centuries there has been a recurrence of various forms of surgery occurring in cycles-becoming fashionable for a period of time and then being buried in oblivion, to be recalled a few hundred years later as a new step in the progress of the surgical attack.

It is only in recent times that we have been able to emphasize that we are able to benefit the vast majority of our patients. Furthermore, we can now offer them a safe operation which, if in itself is not roo per cent. satisfactory, is anyway. non-lethal and helps the vast majority of our patients. In spite of these facts, however, there are still those amongst us who revert to the days of Galen in their enthusiasm to eradicate the varix. Many of such patients come to us at a later date as failures. Their lot, with a history of multiple massive incisions, together with prolonged hospitalization, is a sorry one, and to restore their confidence regarding further treatment is frequently a difficult problem. These patients are apt to approach the clinic with a degree of hopelessness. Many of them have been told by their doctors that nothing more can be done for them, and they need sympathetic handling and reassurance.

The author has for many years noted the more frequent types of operative failure which have presented themselves at a varicose clinic. Those who come to see us usually fall into one of ten categories, although some may present some signs of being in one or more of the groups which we are about to outline. In other words, many cases cannot be definitely placed in a pigeonhole, and all of them require careful examination and assessment in order to determine the reasons why their previous operative t1eatment has met with no success. We now come to the list of the commoner types of surgical ' failure.' 
I. Branches at the sapheno-femoral junction have been missed at the time of the original operation.

This is the most frequent finding and it is not unusual to discover that it is the external superficial circumflex vein which has escaped resection, since this branch is often difficult to define in obese subjects (Fig. I). Another branch which it is only too easy to miss is the accessory saphenous vein which sometimes joins the main saphenous vein deep to the saphenous opening (see Figs. 2 and 3). This vein may often be large and, since it lies on top of and parallel with the femoral vein, may be mistaken for the deep vein when stripping up the saphenous stump. However, it must be recognized that any branch which is missed at the sapheno-femoral junction is capable of bypassing the resected portion of the internal saphenous vein and causing in due course a complete recurrence of the original varicose condition. Patients who suffer from this type of failure usually give the history of a period of definite benefit subsequent to the primary operation, recurrence as a rule appearing within eighteen months.

2. Cases in which the internal saphenous vein has not been ligated at all and the internal superficial femoral vein has been ligated in error.

This error is fortunately not common and it should be noted in the history that these patients show no benefit after operation and that, on examination, the saphenous bulb is usually easily palpable

The majority of those cases in the first two categories, in other words those cases in which tributaries of the sapheno-femoral junction have been missed or that group in which the saphenous vein is still intact, should be given the opportunity of surgical exploration and a further examination of the sapheno-femoral junction. They should be warned that perfect results can in no way be guaranteed, however. With these patients it is usually possible to feel the saphenous bulb either above or below the original scar and, in those who are not too fat, the by-passing branches can also be palpated. These branches may be defined sometimes by the impulse conveyed by coughing. The surgeon would be wise to make a larger incision than is usual when operating on the virgin field and must place the line of his incision either above or below the original scar, choosing whichever position he feels may give him the widest exposure. Special care must be taken, since the old scar tissue may be attached to the femoral vein and tears may result from any rough handling. Once the sapheno-femoral junction has been exposed, a flush ligation and a resection of all adjacent branches should be performed. If

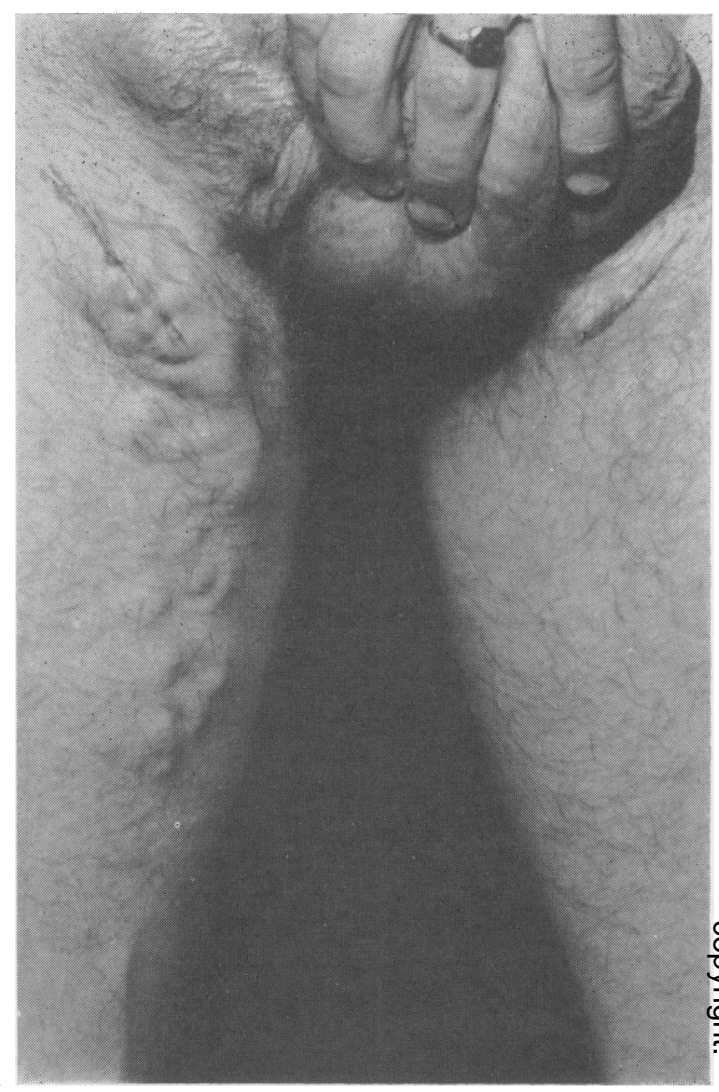

FIG. 1.-Certain branches at the sapheno-femoral junction have not been ligated and a severe recurrence of varices has occurred eighteen months after operation.

thought advisable, a further small incision can be made lower down in the course of the internal saphenous vein so that a nutmeg grater needle may be introduced at this point. Sclerosant may be delivered through this needle if thought to be necessary. Always remember that the usual branches at the sapheno-femoral junction may join the femoral vein high up, going directly into this deep vein. For this reason, when repairing cases of recurrence, it is wise to inspect the femoral vein above the junction with the internal saphenous vein.

3. Those cases in which the external saphenous vein is either incompetent at the time of the original operation or has become so subsequently.

At the time of the first operation on the internal saphenous vein, it is essential to note the condition of the external saphenous vein and to deal with it at the same time if it is found to be incompetent.

Sclerosant therapy may be all that is required with these patients since, as is well known, the 
last inch or so of the external saphenous vein just immediately before it joins the popliteal vein is encased in a sheath of fascia. This fascia may act in much the same way as does a valve and probably to some extent controls the backward pressure of blood in the short saphenous vein. For this reason it is always worth while trying injection treatment before resorting to surgery, especially since recurrences after surgical treatment are common, owing to the difficulty of isolating all the branches at the junction.

4. Cases which have received adequate treatment at the sapheno-femoral junction but in which 'blowouts' (incompetent communicating veins) in the lower third of the leg have either developed since or have not been dealt with at the time of the original operation.

Experience shows that it is usually necessary to resect these 'blow-outs' when they occur in the lower part of the leg at the time that the high resection operation is performed. Similar 'blowouts' in the upper part of the leg and in the thigh can usually be dealt with by means of scarification of the venous intima and do not require separate excisions. Under local anaesthesia these 'blowouts' in the lower third of the leg should be exposed and the communicating veins underlying them should be resected.

5. Cases which develop multiple varices on the inner side of the lower leg after the original operation.

Such cases as a rule need the 'ankle tie' operation and the retrograde injection of a sclerosant, delivered through a plastic tube. This simple procedure may be done painlessly under local anaesthesia. It is important that the tube be withdrawn as the injection is being given, so that the risks of sclerosant entering the deep veins may be minimized (see Fig. 4).

6. Cases which have been operated on correctly for varicose ulceration but who have not been adequately instructed regarding the subsequent prolonged bandage compression treatment which is usually necessary.

The combination of surgery with localized compression treatment is essential for the proper healing of gravitational ulceration and for the removal of the accompanying oedema. If this is not done the frequent result is that the ulcers will heal and then break down at intervals. Advice is needed regarding the correct type of bandage support. With the majority a two-way stretch bandage, correctly applied before leaving bed in the morning, is all that is required in order to remove the oedema and to cause healing. The

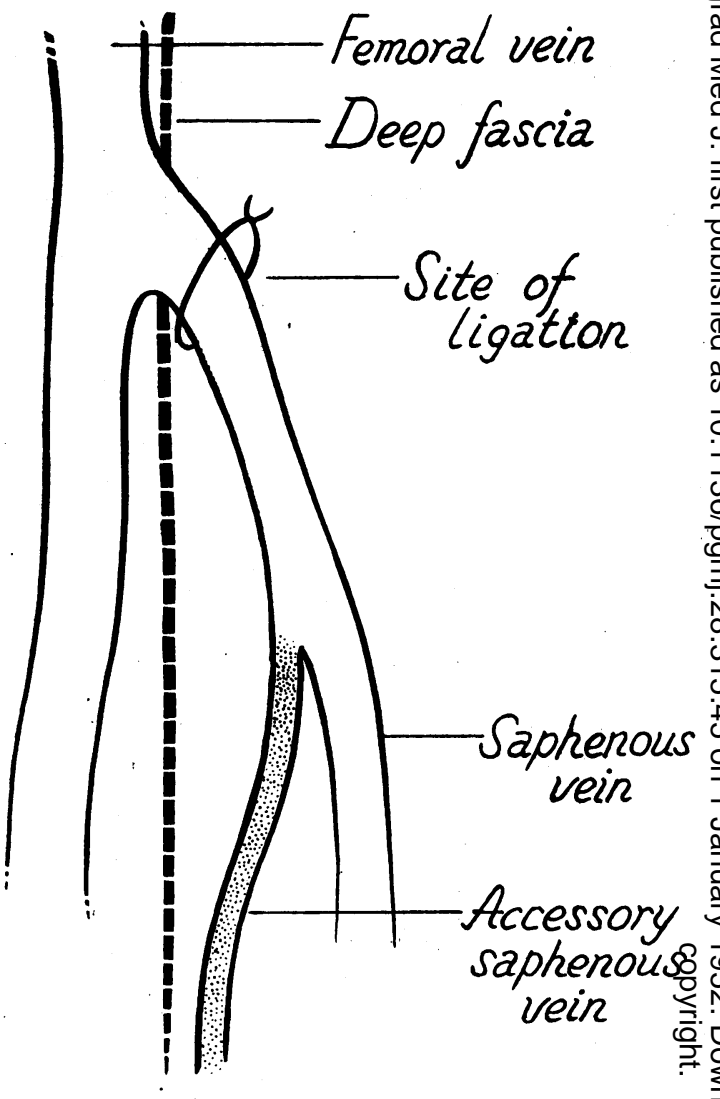

FIG. 2.-The usual method by which the accessory saphenous vein joins the main trunk. This union occurs superficially to the deep fascia and therefore allows of an easy ligation.

bandage should be reapplied during the day and persisted with until a less bulky type of support, such as adequate elastic stockings, may be all that is required for the control of the oedema. For 3 . the less intelligent patients, however, it is far $\frac{\sigma}{3}$ better to use one of the sticky bandages of the Elastoplast type, since the lazy patient may not $\stackrel{\circ}{\circ}$ persist with a two-way stretch bandage once healing has occurred.

7. Cases presenting oedematous legs which have developed shortly after operation.

Such patients are usually suffering from deep vein thrombosis. The causation can be traced 0 either to immobilization of the limb after opera- $\mathbb{W}$ tion, to the use of an over-all excess of sclerosant, to the injection of too much sclerosant into one place, or to the injection being made over a wide- $\mathbb{D}$ open incompetent communicating vein. Sclerosants should only be delivered in small multiple doses, in the right amount and in the right place.

In such cases prevention is far better than cure. 


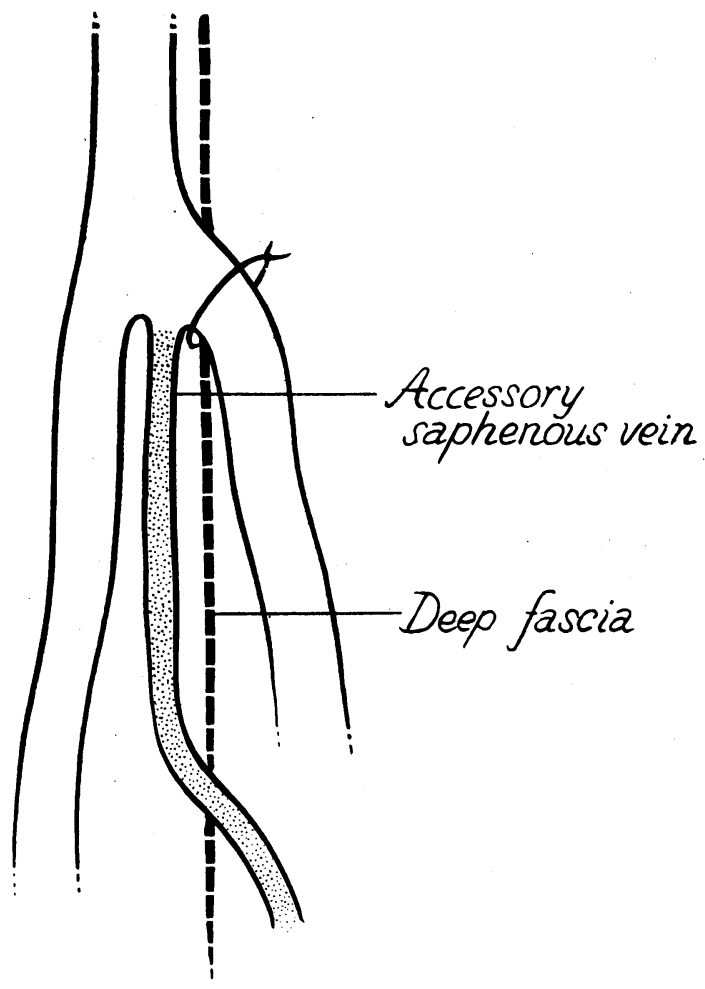

FIG. 3.-A not uncommon method of union of the accessory saphenous vein. In this case the union with the parent trunk is deep to the superficial fascia and the vein lies parallel with the femoral vein. This arrangement at the sapheno-femoral junction may, in some cases, lead to a failure to ligate this branch.

As has already been mentioned, this unfortunate happening can be prevented by an immediate mobilization of the limb after operation. The control in the amount of sclerosant used is of the utmost importance and, in fact, the complete avoidance of all sclerosant is essential in those cases in which the intima of the deep veins has been previously sensitized-as in the case in which a history of white leg is present.

Once the condition has developed, however, it is essential to apply T.W.S. bandages correctly and advise the patient to take plenty of exercise while these bandages are in place. If they are applied so that the pressure is maximum in the lower third of the limb and is relaxed in its upward ascent until it is comparatively loose just below the knee, the oedema will be steadily removed in a majority of cases. Neglect to give active treatment of this type may lead to ulceration or intractable oedema, whereas early treatment will cause the deep veins to take over their full

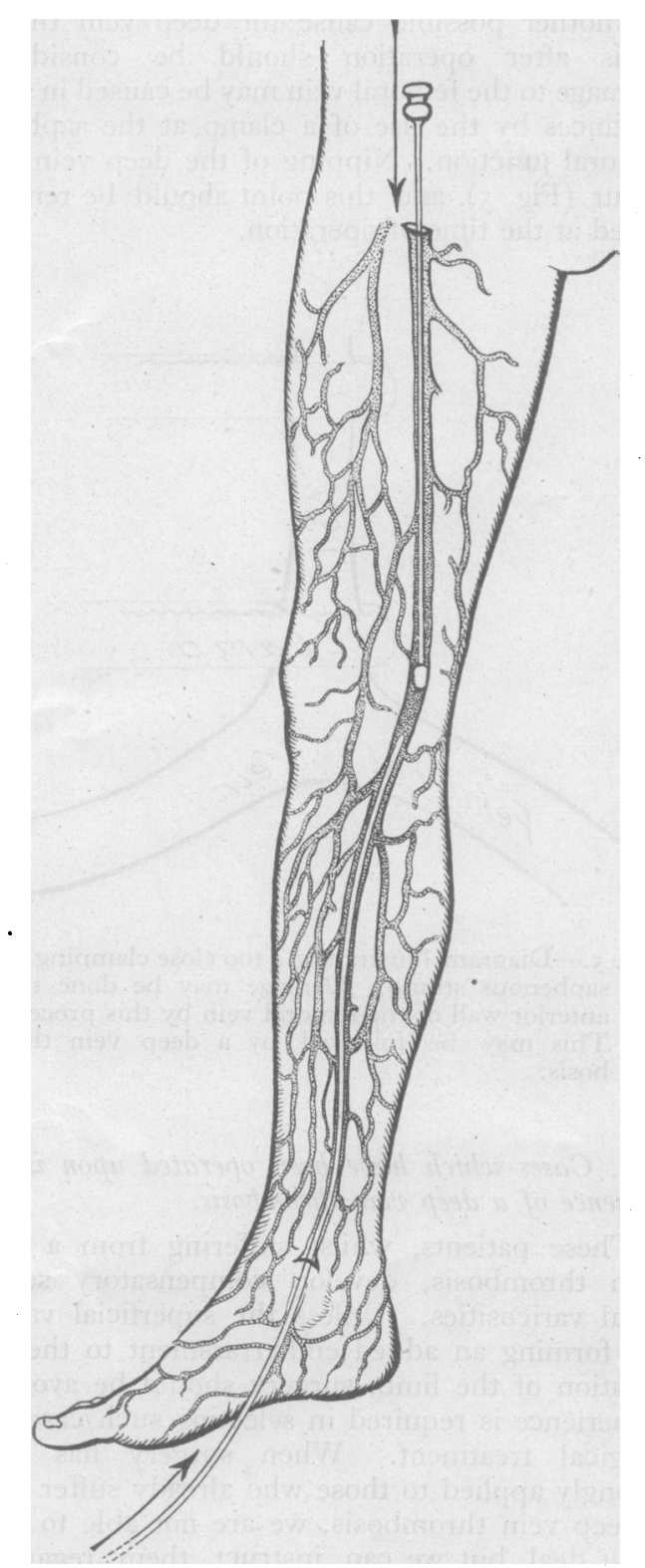

FIG. 4--This diagram represents the author's method of scarifying the venous intima from above by means of a nutmeg grater needle. In addition, there is shown the usual method of performing an ankle tie and introducing a plastic tube through which sclerosant may be delivered. 
work in quite a high percentage of cases. Adequate exercises, physiotherapy and massage are all adjuncts to treatment which should not be forgotten.

Another possible cause for deep vein thrombosis after operation should be considered. Damage to the femoral vein may be caused in some instances by the use of a clamp at the saphenofemoral junction. Nipping of the deep vein may occur (Fig. 5), and this point should be remembered at the time of operation.

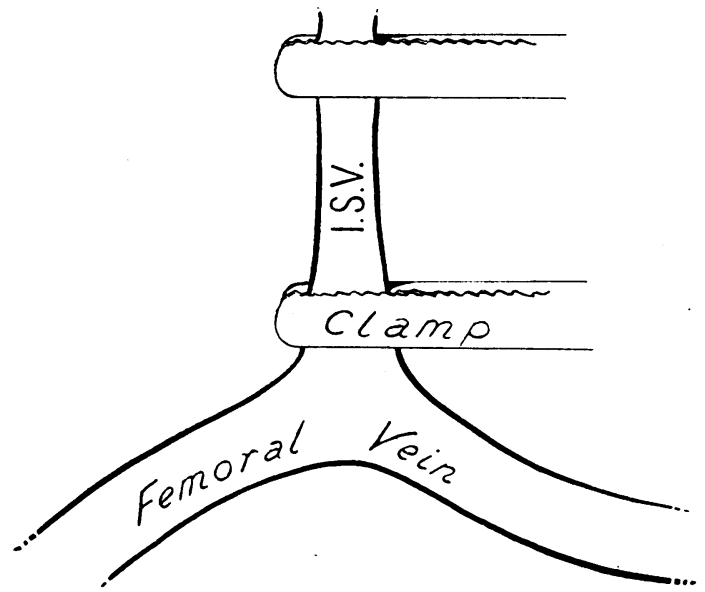

FIG. 5--Diagram illustrating a too close clamping of the saphenous'stump. Damage may be done to the anterior wall of the femoral vein by this procedure. This may be followed by a deep vein thrombosis.

\section{Cases which have been operated upon in the} presence of a deep vein thrombosis.

These patients, whilst suffering from a deep vein thrombosis, develop compensatory superficial varicosities. Unless the superficial varices are forming an added embarrassment to the circulation of the limb, surgery should be avoided. Experience is required in selecting such cases for surgical treatment. When surgery has been wrongly applied to those who already suffer from a deep vein thrombosis, we are not able to do a great deal, but we can instruct them regarding selected exercises, the use of elastic compression bandages and the avoidance wherever possible of all those things which increase the gravitational factor.

9. Persistence of varicose ulceration after adequate surgical treatment, due to the general health of the patient.

It is not uncommon to see persistent ulceration after an efficient surgical procedure. Many of these patients, when examined carefully, may be found to be suffering from a severe anaemia. Correction of this condition will in many cases cause healing to occur. The syphilitic ulcer is now a rarity but tineal infection is always with us and this infection can cause 'failures' to occur after adequate surgical treatment.

I0. That very large group of cases which have not been submitted to a proper follow-up after operation.

All patients should be seen at the least every year after operation-whether they be better or worse. No patients should be promised a cure. They should all realize that varicose disease is a progressive condition and that the surgeon in many cases can but retard this progress. His patients must give him the opportunity of seeing them at regular intervals if their limbs are to be kept in order.

It is not always the fault of the patient that they have failed to attend, since many of them state that they were told that the operation would cure their veins and they are often indignant over the most minor recurrences. Adequate explanation to these patients regarding the progressive character of the varicose state, and the injectior or ligation of branches where necessary, is ofter all that is required. Remember that, when dealing్ with large branches, excision is often less painfur than is the use of a sclerosant. The removal of large branches through small incisions under local anaesthesia is less painful and more lasting in its results.

The purpose of these few comments on our ' failures' is to stimulate interest in their repair and is offered in no critical spirit, since the author of these notes is humbled all too frequently by his own 'failures.' The treatment of varicose veins is an ever-fascinating subject, and 'failures' and their repair add to its interest. Were the fishərman to land a large trout with every cast of his fly, he would soon become bored! No case is too hopeless for us not to be able to offer at least some alleviation. Remember, however, that prior to planning such repair, a careful and thorough examination and assessment of the limbs is essential. A clear conception as to the causes of the original surgical failure is necessary. As in the treatment of all sufferers from varices, the age, physical condition and stresses imposed on the patient by his particular type of work must all receive a careful consideration. Added to this the longevity or outlook regarding the length of life is of importance. The surgical hand should be held when dealing with the elderly who follow sedentary lives. Extensive 
surgery, when all that may be needed is a pair of elastic stockings, is to be condemned. Now that the varicose patient is receiving more specialized treatment in our hospitals, surgical failures are fortunately becoming less frequent. Just as before the days of Robert Jones, fractures were the ' Cinderella' of general surgery, so in our times the varicose patient has received his measure of neglect. The segregation of the varix into a special department in our hospitals is now happening throughout the country and it will not be long before we shall cease to talk of the "failures' of varicose surgery.

\section{Summary}

I. Some of the causes of our 'failures' in the surgery of varices are discussed.

2. There are but few of these cases which cannot be bettered by treatment.

3. A plea for the careful assessment of the cause of each case of failure is made.

4. Some suggestions for the repair of these cases are offered for consideration.

\section{Acknowledgment}

The author is indebted to Messrs. Butterworth for permission to use certain of the illustrations.

\section{BIBLIOGRAPHY} FOOTE, R. ROWDEN (1949), 'Varicose Veins,' Butterworth,
London.

PRATT, G. H. (r946), 'Treatment of Varicose Veins,' 7 . Amer. med. Assoc.s x.32, $70 \mathrm{r}$.

\section{OBSTETRICS \& GYNAECOLOGY}

\section{Postgraduate Medical Journal}

Price : 2s. 9d., post free

Foreword

Sir Eardley Holland, M.D., F.R.C.S., F.R.C.P.

Obstetrics in the National Health Service

Sir William Gilliatt, K.C.V.O., M.D., M.S. (Lond.), F.R.C.P., F.R.C.S.

Modern Views on the Management of Placenta Praevia

C. H. G. Macafee, M.B., F.R.C.S., F.R.C.S.I., F.R.C.O.G.

The Modern Practice of Caesarean SectionSome Controversial Views

S. G. Clayton, M.D., M.S., F.R.C.S., M.R.C.O.G.
Pelvimetry in Obstetrics

H. Cecil Bull, M.R.C.P.

Analgesia and Anaesthesia in Modern Obstetrics G. C. Steel, M.R.C.S., L.R.C.P., D.A.

An Evaluation of the Various Factors in Infertility John Stallworthy, M.A., F.R.C.S., M.R.C.O.G.

The Sympathetic Nervous System in Obstetric and Gynaecological Practice

Albert Davis, M.D., Ch.M., F.R.C.S., M.R.C.O.G.

The Present Position of Radiotherapy in the Treatment of Malignant Disease of the Pelvis M. Lederman, M.B., D.M.R.E.

Published by

THE FELLOWSHIP OF POSTGRADUATE MEDICINE

60, Portland Place, London, W.1 